\title{
Dangerous liaisons: the ecology of private interest and common good
}

Minus van Baalen and Vincent A. A. Jansen

Baalen, M. van and Jansen, V. A. A. 2001. Dangerous liaisons: the ecology of private interest and common good. - Oikos 95: 211-224.

Many ecological interactions that are called mutualistic are in fact mixtures of antagonistic and mutualistic aspects. For example, plasmids exploit their bacterial hosts but also protect them against external threats. In this study, we analyse the conditions for the evolution of what we call 'dangerous liaisons': interactions combining mutualistic and antagonistic aspects. Starting point of our analysis is a model that was proposed as early as 1934 . In this model, partners have to form a complex (either temporary or long lasting) in order to interact. Using this model framework we then set out to define and tease apart private interests of the interacting partners from their common good. This dichotomy provides a unifying perspective to classify ecological interactions. We discuss some examples to illustrate how the outcome of the interaction may depend on densities or on other contextual variables. Finally, we note that having a common good is not a necessary condition for partners to have aligned interests. In a dangerous liaison partners may have interest to cooperate even when this does not bolster the common good.

M. van Baalen, Inst. of Biodiversity and Ecosystem Dynamics, Section Population Biology, Univ. of Amsterdam, P.O. Box 94084, NL-1090 GB Amsterdam, The Netherlands (present address: Institut d'Écologie - UMR 7625, Université Pierre et Marie Curie, Bât. A, 7ème étage CC237, 7 quai St.-Bernard, F-75252 Paris Cedex 05, France [mvbaalen@snv.jussieu.fr]). - V. A. A. Jansen, School of Biological Sciences, Royal Holloway, Univ. of London, Egham, Surrey, UK TW20 OEX.

Samen voor ons eigen

('Together for our own.')

(F. Jacobse \& T. van Es, De Tegenpartij)

Mutualistic interactions occur along the entire range of biological organization and on all timescales, from fleeting encounters to tight symbiosis (Bronstein 1994a, Leigh and Rowell 1995, Frank 1997, Herre et al. 1999). Such interactions are often thought to have arisen out of parasitic (or more generally, antagonistic) interactions. If it is true that such evolutionary transitions have occurred repeatedly and on many levels of biological organization, there must be common underlying principles that are independent of much of the biological detail. However, as Herre et al. (1999) note, at present there is no such general theory, and the problem 'revolves around explicitly defining vague terms such as "alignment of interests", (see also Leigh and Rowell 1995, Leigh 1999). In this article, we will discuss a general framework in an attempt to fill in this gap.

Despite the fact that ecological interactions are often described as either competitive, antagonistic or mutualistic, many interactions are actually a mixture of these aspects. Many parasites confer benefits to their hosts, while mutualists may have detrimental effects on their partner. It is increasingly recognized that any interaction is (potentially at least) a mixture of conflicting and overlapping interests. The net outcome (at the population level) is then just the end to which the balance is tipped.

This balance may be delicate. For example, lysogenic phages can carry resistance genes against antibiotics which, upon infection, endow a bacterial host with

Accepted 3 May 2001

Copyright (C) OIKOS 2001

ISSN 0030-1299

Printed in Ireland - all rights reserved

OIKOS 95:2 (2001) 
antibiotic resistance (Stewart and Levin 1984). Both the phage and the host will benefit from this resistance if their environment contains antibiotics; then, partners have a clear common interest. On the other hand, the phage still is a parasite that will exploit and may eventually kill its host. Such an interaction may be called a 'dangerous liaison' (Haig 1997): there is a benefit associated with teaming up, but a substantial risk as well. Whether the host should tolerate its parasite depends on the presence of antibiotics. As long as partners have a sufficient common interest, they should continue to cooperate, but as soon as conditions change to boost selfish interests, one (or both) of the partners may defect and a struggle rather than a harmonious relationship ensues.

Leigh and Rowell (1995) and Leigh (1999) point out that the crucial aspect of the evolution of mutualism is whether partners have a sufficient common interest. If common interests prevail over selfish interests, a partner will do better to invest its resources for the common good. The difficult part, however, is to determine how, in a given interaction, such a common interest arises, and how it may be bolstered (or erode) in the course of evolution.

To understand under what conditions a common interest arises, and how it may eventually take precedence over the partner's selfish interests is a central problem in biology. What is called an 'individual' is often, in fact, an association of lower-level entities that have, or have had in the past, an independent existence (Margulis 1970, Maynard Smith and Szathmáry 1995). Eukaryotic cells contain mitochondria and other organelles that are demonstrably evolutionary vestiges of independent organisms. On a higher level, lichens are formed by a symbiosis of fungi and photosynthetic algae (Sapp 1994). On a lower level again, it can be said of many genetic entities, such as plasmids, transposable elements, and segregation distorters, that their fate is not tightly linked to that of their carrier. In fact, the same can be said of 'ordinary' genes, as is demonstrated by numerous examples of 'selfish genes' and the resulting intragenomic conflicts (Dawkins 1976, Hurst 1995 , Pomiankowski 1999). Before the question can be answered under what conditions an entity renounces its evolutionary sovereignty, there must be a reason for partners to associate and contribute in the first place.

To address questions like these, the definitions of species interactions (competition, antagonism - the category that includes parasite-host interactions but also predator-prey interactions - and mutualism) must be made more precise. The classical definitions are based on ecological principles, the effect of populations on one another. In practice they are usually derived from the sign structure of the community matrix, whose elements ('interaction coefficients') give the net effect of one population on the growth rate of another. If the per-capita growth of a species $A$ increases with the density of a species $B$ and the same is true vice versa, then species $A$ and $B$ are said to be mutualists. In contrast, if the interaction coefficients are of opposite signs, one of the species 'preys' on the other, and the interaction is said to be antagonistic (see, e.g., Cheng 1991). According to these definitions the interaction between phage and bacterial host would be classified as antagonistic, regardless of a (possibly small) beneficial effect of the phage's resistance genes. A consequence of such an effect-based classification is that an interaction is either parasitic or mutualistic. These definitions thus capture only the net outcome of what may actually be a quite delicate balance. To make this balance appear one must therefore change focus from the level of the population to that of the individuals composing the system.

That an individual host and its parasites share a common interest is intuitively clear: if the host dies, the parasite loses a carrier for transmission to other hosts. Similarly, a plant and a pollinator share a common interest: for both it is important that their interaction works out as it should, as reproduction is at stake for both. Less intuitively, perhaps, but we can speak of the common interest between predator and prey. Yet how to explain, for example, the evolution of signalling conventions between predators and prey? A well-known example is that of gazelle 'flagging', and other so-called 'predator deterrent' behaviours (Caro 1995, Caro et al. 1995). Gazelle present conspicuous signals when they perceive themselves as a possible target of a predator. The function is thought to be to dissuade the predator from pursuing the attack. Obviously, the prey individual gains when the predator does not kill it. But for the signalling system to evolve, there must also be something in it for the predator. And in fact, the predator individual gains a costly chase when it can infer that it will be unsuccessful. Presumably, the predator will then devote its attention to less agile prey, which may even confer a second, indirect advantage to the signaller: the information may induce the predator to attack a competitor. Moreover this example suggests that the benefits to both partners are context dependent: the attacker may ignore the message if there are no weaker prey around. Prey-to-predator communication may therefore only evolve in systems where there is sufficient competition among the prey. The subtle mixture of common interest in the interaction will be completely missed in any classification based on the effects of populations on one another. Thus, the evolution of prey-to-predator signalling requires alignment of interests at the individual level, but if there is a case of a dangerous liaison this is one!

Whenever two individuals interact, there may be scope for cooperation even if the interaction is antagonistic on the whole. Conversely, even in a mutualistic interaction, selfish behaviour may occur. From a theoretical perspective, the problem is how to disentangle 
selfish and common interests of interacting individuals. How does one assess the common interest of a prey and a predator engaged in a chase, for example? How does this common interest depend on the context? It will be advantageous for a host to tolerate a mild parasite only if there are more dangerous parasites around. As another example, it may pay for a prey to signal its capacity for escape only if there are weaker individuals around. Much of the incentive for cooperation might depend on the intensity of within-trophic level competition, on the principle of 'the enemy of my enemy is my friend'.

In this article, we will carry out an ESS analysis of the interactions between individuals of two populations. The structure of this article is as follows. First we will discuss how a range of interactions from outright competitive to fully mutualistic (from competition for resources to predator-prey and host-parasite interactions, via client-provider to obligate symbiosis) can be expressed in terms of a common framework. Then we will outline how fitness is defined in this framework (from the invasion exponent of rare mutants, see Metz et al. 1992, Rand et al. 1994, Dieckmann and Law 1996, Geritz et al. 1997), and how this concept can be used to sharpen the definitions of antagonism and mutualism. These fitness concepts can then be dissected to separate selfish interests from the common good of the interactants. Finally, we will analyse the conditions for alignment of interests and how this is related to private interest and common good. We intersperse the development of our argument with a number of examples to discuss the salient points. To preserve the flow of the argument most of the mathematical detail is referred to appendices.

\section{Interacting individuals}

Models for populations of interacting individuals date back as far as 1934 (Kostitzin 1934, Wolin 1985). Kostitzin's approach has resurfaced many times and in many guises, but always in relation to questions about conditions favouring association (Law and Dieckmann 1998, Yamamura 1993, 1996, Genkai-Kato and Yamamura 1999). Law and Dieckmann (1998) derive the model from considerations of physiological interactions between unicellular organisms; Yamamura and colleagues (Yamamura 1993, 1996, Genkai-Kato and Yamamura 1999) used it to study the evolution of vertical transmission in host-parasite interactions. Depending on the parameters, the mathematical framework can model other types of interactions as well, including predator-prey and client-provider interactions such as between plants and pollinators. So-called 'marriage models', used to assess the rate of spread of sexually transmitted diseases, are another example of this class of models (Heesterbeek and Metz 1993).
The underlying principle of the framework is that interactions between individuals (denoted $x$ and $y$ ) take place only when two individuals associate and form a complex $x y$, see Fig. 1A. Such associations may last as long as no partner dies, as in some host-parasite interactions, but it may also be of short duration, as in the case of predator-prey interactions, where a predator pursuing a prey may be considered a temporary (or virtual) predator-prey association.

The framework is given by three differential equations that govern the densities of free individuals and of the complex,

$$
\begin{aligned}
& \frac{\mathrm{d}[x]}{\mathrm{d} t}=F_{x}[x]-\beta[x][y]+P_{x}[x y] \\
& \frac{\mathrm{d}[y]}{\mathrm{d} t}=F_{y}[y]-\beta[x][y]+P_{y}[x y] \\
& \frac{\mathrm{d}[x y]}{\mathrm{d} t}=\beta[x][y]-M_{x y}[x y],
\end{aligned}
$$

where symbols enclosed in square brackets denote densities of free individuals and of complexes, and $\beta$ the rate of encounter of free $x$ and $y$ individuals. The other symbols represent fitness components of free and bound individuals (see also Fig. 1B). The terms $F_{x}$ and $F_{y}$ denote the net rate of reproduction of free individuals,

$F_{i}=\rho_{i}-\mu_{i}$

where $\rho_{i}$ denotes the rate of reproduction of free individuals of species $i$ and $\mu_{i}$ their mortality rate. Because the contribution of the association is not included, we will call the rates $F_{x}$ and $F_{y}$ the 'free fitness component' of free $x$ and $y$, or $x$ and $y$ 's 'free fitness' for short.

Once an $x$ - and a $y$-individual have formed an association, this association may persist for a certain time, producing free $x$ and $y$ and possibly new $x y$ complexes. We can define the net mortality rates of such complexes as
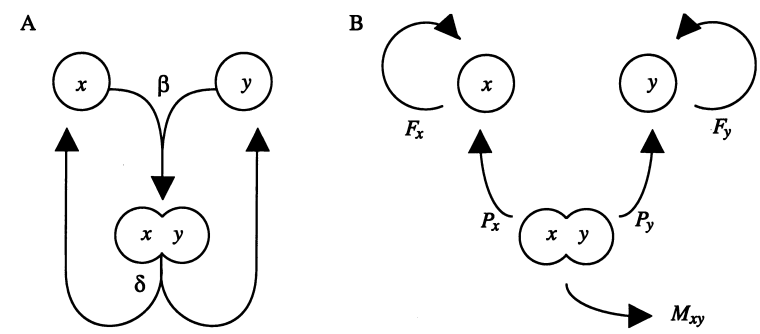

Fig. 1. Schematic representation of the processes affecting free and bound individuals. (A) Individuals of species $x$ and $y$ can combine to form interacting complexes $x y$ (with encounter rate $\beta$ ), which can then dissociate again (with rate $\delta$ ). (B) Fitness components of free and bound individuals (for an explanation of the symbols see text). 
$M_{x y}=v_{x}+v_{y}+v_{x y}+\delta-\sigma_{x y}$

where $v_{x}$ and $v_{y}$ denote the uncorrelated mortality rates (one of the partners dies), $v_{x y}$ the correlated mortality rate ( $x$ and $y$ die simultaneously), $\delta$ denotes the rate of dissociation and, finally, $\sigma_{x y}$ denotes the rate of joint reproduction of $x$ and $y$ individuals (such that new $x y$ complexes are produced). Note that $M_{x y}$ is not a true mortality rate as it incorporates a rate of reproduction $\left(\sigma_{x y}\right)$, but in what follows the results are most easily understood if it is interpreted as such.

The third class of fitness components are the 'private interests' of individuals in association. Whenever the $y$ partner dies and whenever a complex dissociates, a free $x$ individual is produced again. This, and the independent birth rate of the partners $\sigma_{i}$ (i.e., the rate of production of free offspring) contributes to the dynamics of free individuals, and is represented by $P_{x}$ and $P_{y}$,

$P_{x}=\sigma_{x}+v_{y}+\delta$

$P_{y}=\sigma_{y}+v_{x}+\delta$.

In order to prevent unchecked population growth, some density dependence needs to be introduced. Density dependence may act in various ways, but for the general framework it is irrelevant which of the demographic rates (the rates of reproduction, mortality, association and dissociation) are density dependent. We will discuss the consequences of various forms of density dependence later on.

As we show in Appendix A, which type of ecological interaction the framework represents depends on the relative values of the parameters. This means that by letting parameters evolve, we can study transitions from one kind of interaction to another. Evolution of parameters can be analysed using evolutionary game theory (Metz et al. 1992, Rand et al. 1994, Dieckmann and Law 1996, Geritz et al. 1997), where the goal is to work out how parameter combinations will evolve and at which evolutionary attractor they will eventually end up. (An evolutionary attractor, as its name suggests, attracts all evolutionary trajectories and is thus the dynamical equivalent of such well-known concepts as unbeatable strategies (Hamilton 1967) and evolutionarily stable strategies (ESS) (Maynard Smith and Price 1973, Maynard Smith 1982).) An essential element in this approach is the set of constraints that delimits combinations of parameters. The physiological considerations that underlie Law and Dieckmann's (1998) model give rise to such constraints. Here we will outline how this framework can be analysed for any set of constraints. More specific examples will be worked out in appendices.

Consider a resident system in equilibrium. When can a mutant (of either population) invade? In principle the mutant's traits may affect all of its life-history parame- ters, free and in association. Indicating the mutant's life-history parameters by hats, we may therefore have $\hat{\rho}_{x} \neq \rho_{x}, \quad \hat{\mu}_{x} \neq \mu_{x}, \quad \hat{\sigma}_{x y} \neq \sigma_{x y}$, etc. Adding such a mutant to the system requires not one extra differential equation but two: one for mutants that are free and one for those that are in association with a (resident) $y$ individual. From invasion analysis (see Appendix B) a fitness measure can be derived that takes both states into account,

$W_{x}=\hat{F}_{x}+\hat{\beta}[y] \frac{\hat{P}_{x}-\hat{M}_{x y}}{\hat{M}_{x y}}$.

This quantity has the same sign as invasion fitness: if it is positive, the mutant will invade, if it is negative, the mutant will go extinct. The first term represents the contribution of the free state to the mutant's overall fitness, the second term gives the contribution of the association. The latter is the product of the probability of association $(\overline{[y]}$ denotes the equilibrium density of available partners which is set by interaction between the $y$ population and the resident $x$ population) and the number of offspring that are born during the association. If there is no correlated reproduction $\left(\sigma_{x y}=0\right)$ the quantity $1 / \hat{M}_{x y}$ is just the expected duration of an association. During this time offspring (free and bound) are produced with rate $\hat{P}_{x}-\hat{M}_{x y}$. An expression structurally similar to Eq. (6) results for the fitness of mutants of the $y$-type:

$W_{y}=\hat{F}_{y}+\hat{\beta}[x] \frac{\hat{P}_{y}-\hat{M}_{x y}}{\hat{M}_{x y}}$.

For brevity, we will discuss the interaction from the point of view of $x$-type individuals, but an analogous analysis can and should be carried out, mutatis $m u$ tandis, for the $y$ population.

One can get a more intuitive idea by considering what these fitness components represent in a host-parasite setting. $F_{x}$ represents the net rate of reproduction of uninfected hosts (if $x$ is the host) and $F_{y}$ the net rate of reproduction of parasite propagules (the free state). When parasite propagules do not reproduce on their own, as is often but not always the case, $F_{y}$ will be negative. $M_{x y}$ represents the net rate of mortality of infected hosts (net, because it is corrected for vertical transmission); $P_{x}$ represents the rate of production of uninfected host offspring; $P_{y}$ represents the rate of production of parasite propagules.

\section{Ecological interactions}

In ecological modelling, usually no distinction is made between the interaction between individuals of two species and the interaction between two populations. 
The first important thing to realize is that these do not necessarily amount to the same. Two populations may interact without individuals of the two populations ever coming into contact. For example, competition for a common resource would cause density dependence in reproduction or mortality rates. Many ecologically important interactions, however, are between individuals of the two species: killing, parasitizing, helping, communicating, etc., all these are about one individual dealing with another. For these cases, individual-level definitions are more appropriate.

So let us first characterize the interaction from the point of view of the interacting individuals, as opposed to the interaction at the level of populations. Here we will use the fact that for an individual of species $i$ it is advantageous to be in association if 'bound' fitness $\left(P_{i}-M_{x y}\right.$, see Fig. 1B) is larger than free fitness $\left(F_{i}\right)$ as can be deduced from fitness measure (6). This we can do for both partners. If it pays both to be in association then one can speak of mutualistic interaction. Would it pay one of the partners to be in complex with the other, whereas the other prefers to be in the free state, then we can speak of an antagonistic (parasitic) interaction. And finally, the interaction can be said to be competitive when the interaction is disadvantageous for both partners

To make this more concrete, consider the hypothetical example of two prey populations that share a common predator (this example is worked out in more detail in Appendix C). In absence of predation, there are only disadvantages of being in association, but it is assumed that complexes are better protected against predator attack. If predation pressure $(p)$ is low, the interaction is essentially competitive, selection on both species is to avoid the association. If predation pressure increases, however, the protection offered by the association will outweigh the costs for one of the species, but not for the other (compare Fig. 2A and B). Then, the interaction has become antagonistic: one species profits from the other but not the other way round. Eventually, when predation pressure becomes even higher, it becomes profitable for individuals of both species to seek partners to associate with; this is a case of mutualism. An example of such a transition is provided by Omacini et al. (2001) who discuss an example where plants infected with endophytes are better protected against herbivores. Then, the interaction changes from parasitism to mutualism when the density of herbivores increases.

It is important to realize that these definitions are different from the interaction coefficients of the two species, the signs of which are usually taken as characterizing species interactions (Cheng 1991). The difference is that there may be all sorts of density dependence in the demographic parameters, that we have not yet taken into account. This becomes evi-

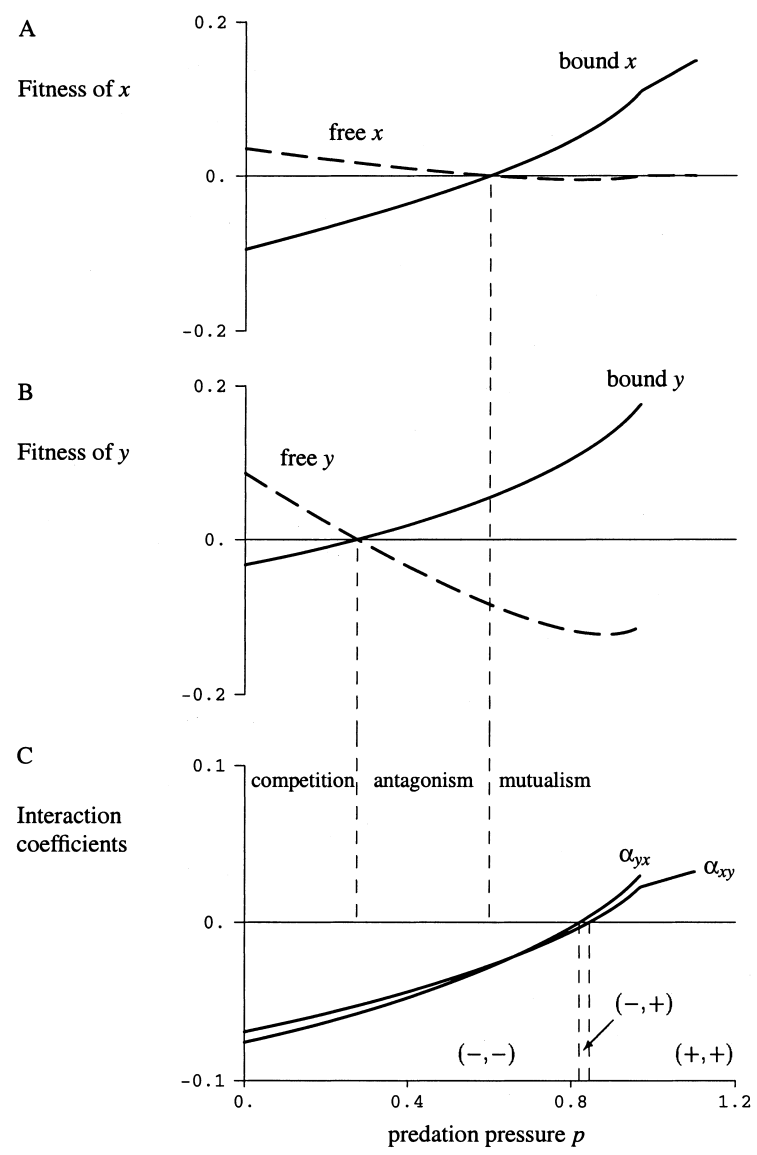

Fig. 2. Effects of common defence on fitness components and population-level interaction as a function of predation pressure $p$. (A) Free $\left(F_{x}\right)$ and bound fitness $\left(P_{x}-M_{x y}\right)$ of species $x$, (B) free $\left(F_{y}\right)$ and bound fitness $\left(P_{y}-M_{x y}\right)$ of species $y$, and (C) interaction coefficients of populations $x$ and $y$. Also indicated are the signs of the interaction coefficients. The definitions of the model can be found in Appendix C; parameter values used to draw the plot: $r_{x}=r_{y}=2, \kappa_{x x}=\kappa_{y y}=0.01$, $\kappa_{x y}=\kappa_{y x}=0.005, \mu_{x}^{0}=0.9, \mu_{y}^{0}=1.1, \beta=0.5, \delta=1, C_{x}=0.2$, $C_{y}=0.1, B=0.5$.

dent if we calculate the overall per-capita effect of both species on one another, that is, if we calculate the elements from the community matrix. Fig. $2 \mathrm{C}$ reveals a similar pattern from competition to mutualism when predation pressure increases, but not at the same threshold densities. This is not surprising, as the interaction coefficients incorporate the effects of niche overlap between both species as well. Thus, even when at the population level $x$ and $y$ compete, at the individual level they may mutually benefit.

When we express fitness measure (6) in the form

$W_{x}=\hat{F}_{x}+\hat{\beta}[\bar{y}]\left(Q_{x}-1\right)$ 
we see that per association one free $x$ disappears while $Q_{x}=\hat{P}_{x} / \hat{M}_{x y}$ free offspring are produced. The ratio $Q_{x}$ thus measures the net profitability of being in association. If $Q_{x}$ is larger than one the association is advantageous for $x$, if it is smaller the association is detrimental.

\section{Common good and private interests}

As discussed before, partners may cooperate without being engaged in a full-blown mutualistic interaction. Here, we will put this notion on a more concrete footing. That is, we will use the fitness expression we derived earlier to define the 'common good' of both partners and their 'private interests'. This allows us to address the question of how an individual should strike the balance between the two fitness components.

Comparison of the expressions describing the fitness of $x$ and $y$ individuals (Expressions (6) and (7)) shows that apart from the association constant $\beta$ the only common element in $W_{x}$ and $W_{y}$ is the association's persistence $1 / M_{x y}$; all other components differ. The common good can therefore be defined as the association's persistence: promoting persistence benefits both partners. The private interest of partner $x$ is then simply $P_{x}$, which is indeed promoted by such aspects as (1) increasing selfish reproduction, (2) killing the partner, and (3) dissociating from the partner.

What options does a mutant of species $x$ have to increase its fitness? Inspection of Expression (6) relating all fitness components of $x$ allows the following observations. All else equal, it pays to increase free fitness $F_{x}$, which is obvious. Similarly and equally obvious, all else equal it pays to increase private interests $P_{x}$. And finally, again all equal, it pays to increase the complex' survival (decrease $M_{x y}$ ): when the complex survives the partners survive too. However, it may be impossible to accrue all these benefits at the same time. For example, shifting resources from the production of coupled offspring $\left(\sigma_{x y}\right)$ to the production of free offspring $\left(\sigma_{x}\right)$ increases private interest $P_{x}$ but decreases complex persistence $1 / M_{x y}$ at the same time. In a similar vein, increasing $1 / M_{x y}$ by reducing the rate of separation $\delta$ decreases $x$ 's private interest as well. In addition, constraints will link traits to various life-history parameters, so that any trait is likely to have an effect on private interest $P_{x}$ and on common good $1 / M_{x y}$ at the same time. When both are positively dependent on the trait there is no problem, but more often than not there will be a negative relationship: promoting private interest is likely to damage the common good.

So how should a mutant strike the balance between private interest and common good? From Eq. (8) it can be deduced that a mutant attempting to maximize its fitness should maximize the ratio
$\hat{Q}_{x}=\frac{\hat{P}_{x}}{\hat{M}_{x y}}$

when optimizing a trait that affects $\hat{P}_{x}$ and $\hat{M}_{x y}$ but not $\hat{F}_{x}$ or $\hat{\beta}$.

If there is no joint reproduction, $Q_{x}$ is the expected number of free $x$ individuals produced per complex during its existence. It gives an indication of the overall profitability of the interaction for an $x$ individual: if it is larger than unity, $x$ will accrue a net benefit from the interaction. The important thing to notice is that profitability $Q_{x}$ can be increased by increasing private interests but also by favouring the common interest.

Consider a small change in a trait $c_{x}$ that increases common good (decreases $\hat{M}_{x y}$, i.e., $\left.\mathrm{d} \hat{M}_{x y} / \mathrm{d} \hat{c}_{c}<0\right)$. Such a change is favoured if $\mathrm{d} \hat{Q}_{x} / \mathrm{d} \hat{c}_{x}$ is positive, that is, if

$\frac{\mathrm{d} \hat{P}_{x}}{\mathrm{~d} \hat{c}_{x}}>Q_{x} \frac{\mathrm{d} \hat{M}_{x y}}{\mathrm{~d} \hat{c}_{x}}$.

Of course, such a trait will be favoured if it also favours private interest (then $\mathrm{d} \hat{P}_{x} / \mathrm{d} \hat{c}_{x}$ is positive while the righthand side of the inequality is always negative). But promoting the common good may be favoured even when it harms $x$ 's private interest. The optimum strategy for the mutant is that strategy that renders left- and right-hand sides equal. Such optimum strategies can be found by graphical methods.

The concepts of private interest and common good lead to a number of quite general observations. First of all, the magnitude of a partner's private interest relative to the common good gives an indication of the importance of the association for a partner. That is, if a partner's private interest $P_{x}$ is larger than $M_{x y}$ it reaps a net benefit from the association $\left(Q_{x}>1\right)$. If not, being in association confers a net disadvantage. Second, the magnitude of private interest gives an indication of the evolutionary independence of a partner. That is, if $P_{x}$ decreases, $x$ 's fitness becomes more and more dependent on the common good of the partnership. Would $P_{x}$ eventually attain zero, then $x$ will have completely renounced its own independent existence. As soon as $x$ has some independent reproduction $\left(\sigma_{x}>0\right)$, if death of the $y$-partner does not inevitably lead to the death of the $x$ partner as well $\left(v_{y}>0\right)$, or if there is separation of partners $(\delta>0)$, inevitably $x$ has a definite private interest. Third, private interest relative to common good gives an indication how selection is likely to operate on both. This, however, goes in the opposite direction of what one might naively expect. That is, the smaller the private interests the more important they are and hence the larger the selection pressure to be selfish.

In a classical host-parasite system, for example, where the parasites can vary their virulence and the hosts can vary the allocation of energy and resources to fight the parasite once infected, host and parasite have 
a clear common interest in the survival of the host, as has been often observed. However, to the parasite, this common interest is more important than it is to the host! Fig. 3 shows a pair of coevolutionarily stable strategies (CoESSs) in a host-parasite interaction. The parasite curve represents the effects of variations in virulence on the parasite's private interests and the common good of the association. As can be seen, at the CoESS $\hat{Q}_{y}=\hat{P}_{x} / \hat{M}_{x y}$ is maximal. This represents the optimum solution to the classical dilemma of increasing transmissibility (private interest) and host longevity (common good). The host's curve results when the host varies the intensity with which it fights the parasite. For the host, selection pressure (indicated by the arrow) points more in the direction of increasing private interests; in other words, to the host, private interests should prevail. For the parasites, in contrast, the common good is relatively more important, the selection pressure (arrow) indicates that to the parasite it pays more to decrease $M_{x y}$ than to increase $P_{y}$. Note that the direction of selection pressure is related to the overall profitability of the association: the parasite's curve is above the dashed line in Fig. 3, the host's below.

In the host-parasite example that we discussed, the emergence of a common good can be discussed without

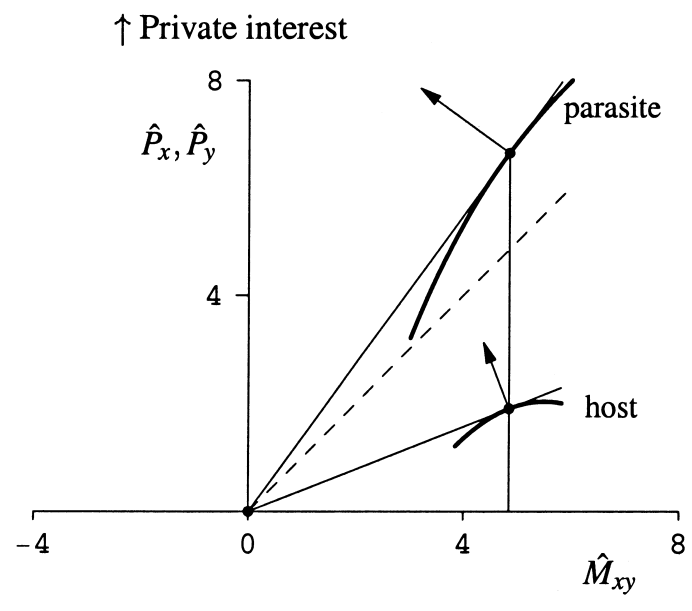

\section{$\longleftarrow$ Common interest}

Fig. 3. An example of the balance of private interests and common good in a host-parasite interaction. As explained in the text, mutants should increase their private interest $P_{i}$ but at the same time decrease the association's mortality $\hat{M}_{x y}$. The optimum ratio between the two is such that the tangent passes through the origin. The arrows indicate the direction of selection. Note that the CoESSs for host and parasite will coincide at the same value for $M_{x y}$. Constraints assumed to draw this plot (the CoESS was found numerically): the parasites have a virulence trait $\hat{c}_{y}$ that links propagule production, $\hat{\sigma}_{y}=4 \hat{c}_{y}$ / $\left(1+0.25 \hat{c}_{y}\right)$ to the mortality rate of infected hosts, $\hat{v}_{x y}=1+$ $\hat{c}_{y}$; infected hosts have a defence trait $\hat{c}_{x}$ that links their rate of reproduction, $\hat{\sigma}_{x}=1.2\left(1-\left(\hat{c}_{x} / 2\right)^{2}\right)$ to recovery rate (parasite death), $\hat{v}_{y}=\hat{c}_{x}$. No vertical transmission is assumed in this example. Above the dashed line, being in association confers a net benefit, below a net disadvantage.
A

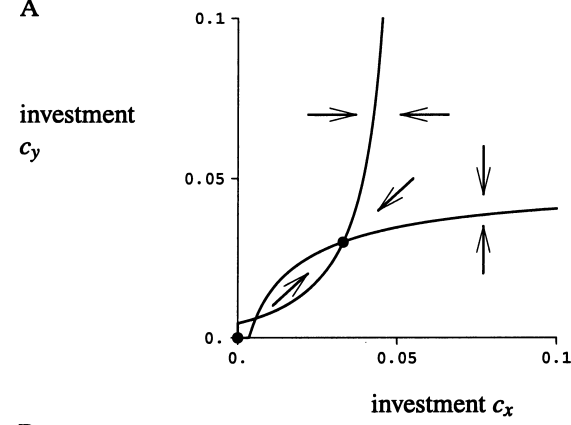

B

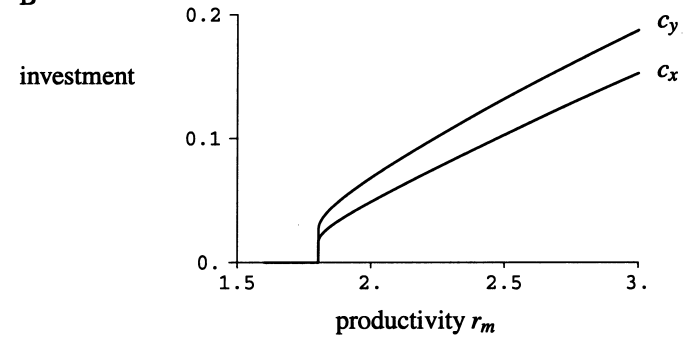

C

density

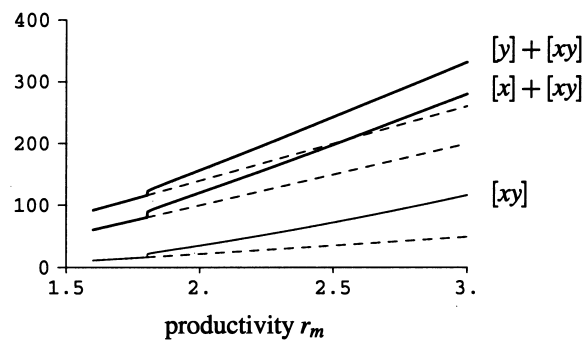

Fig. 4. The evolution of mutually assisted competition. (A) Selection isoclines for the investment of partners (expressed as a fraction of their potential rate of reproduction, $c_{x}, c_{y}$ ) helping each other to survive intraspecific competition. CoESSs are indicated by the heavy dots. Panel (B) shows the proportion of investments under the mutual-aid CoESS, panel (C) the densities under the mutual-aid CoESS (drawn lines) and under the no-mutual-aid CoESS (dashed lines), as a function of the productivity of the environment $\left(r_{m}\right)$. Both partners have equal demographic parameters, with the exception that the rate of reproduction of $y$ is always $20 \%$ greater than that of $x$. (The model is described in Appendix D. Parameters: $\rho_{x}=r_{m}, \rho_{y}=1.2 r_{m}, m_{x}=m_{y}=1, \kappa_{x}=\kappa_{y}=0.01$, $\varepsilon_{x}=\varepsilon_{y}=30, \beta=0.01$.)

considering the external world. Often, however, the benefits of association are strongly density dependent. For example, plants and soil microorganisms may engage in mutually beneficial relationships in which they exchange vital resources (Parker 1999). One effect of such exchanges is that it helps partners compete. Indeed, the model analysed in Appendix D shows that such mutual aid may be a CoESS (Fig. 4A), but that it is only favoured when the productivity of the environment exceeds a certain threshold value (Fig. 4B). Mutual aid only pays when densities, and thus the intensity of competition, are sufficiently high (Fig. 4C). Note that this example is entirely based on the assumption 
that partners in association can withstand competition more efficiently. Of course, in other cases partnerships may be based on resistance to adverse conditions or other factors. In those cases, the reasons for teaming up will be the same but they need not be density dependent in the same way (see also Hochberg 2000).

\section{Alignment of interests}

We have shown that, under certain conditions, individuals will sacrifice part of their private interest for the common good, the common good being defined as the survival of the association. This trade-off may explain such phenomena as reduction in virulence in host-parasite systems and the exchange of resources to help partners compete. Such cooperation works because promoting the common good serves both partners.

However, this does not explain all forms of cooperation. For example, a predator chasing a prey does form a temporary association with the prey individual. However, it is hard to imagine why they both should attempt to prolong the time they are in pursuit. Yet behaviour like 'stotting' in gazelle is commonly explained as a way of communication between pursuer and pursued (Caro 1995, Caro et al. 1995). Such communication would not evolve if there were not anything 'in it' for both. But as bolstering the common good (prolonging the pursuit) cannot be of mutual advantage, how should we explain such forms of cooperation?

As it turns out, bolstering the common good is only one of the conditions favouring cooperation. In fact, the key concept here is 'alignment of interest' which is not the same as common good. Our framework allows a definition of this commonly used concept: alignment of interest occurs when the fitness of both partners increases with a given change. For example, when being in association is unprofitable to both partners, both will let their private interest prevail over the common good. Yet this does not preclude overlap in interest: in fact both have an interest in shortening the interaction, so whatever one partner does to achieve this will benefit the other as well. In other words, there is an alignment of interests in that partners 'agree' to sacrifice the common good, rather than bolstering it. It is as if the interaction is actually a tripartite game: both partners team up against the partnership! Under such conditions individuals may be selected to sacrifice some of their resources to a cooperation to stop the interaction.

Thus, cooperation through alignment of interests is not the same as serving the common good. By comparing the amount by which the partners' fitnesses increase or decrease with changes in the different demographic parameters, we can determine where their interests are aligned and where they are in conflict. This is worked out in Appendix E and the results are summarized in Table 1. As can be seen, an arbitrary interaction may be a mixture of aligned and conflicting interests. Note that Table 1 presents the fitness effects of changes in only a single demographic rate. In general, a trait will affect (through constraints or trade-offs) more than one demographic parameter at the same time and under those circumstances alignment depends on a weighted mixture of all effects. This means that conditions for alignment of interest may be not at all immediately obvious. Detailed knowledge of the interaction may then be necessary to work out the overall effect.

\section{Discussion}

That ecological interactions are often complex mixtures of conflicting and overlapping interests is increasingly being recognized by evolutionary biologists (see reviews by Bronstein 1994a, b, Leigh and Rowell 1995, Frank 1997, Herre et al. 1999, Keller 1999). This insight is corroborated by our analysis which suggests that cooperation may evolve under different scenarios and in widely different settings.

The first scenario is that individuals sacrifice some of their private interests to serve the common good. An example of this scenario would be the interaction between plants and soil microorganisms. Such an association is sufficiently profitable for both partners (when their competitive ability is boosted compared to freeliving individuals) that it warrants an investment to promote the partnership. A second scenario, however, is entirely opposite: if the interaction is detrimental for both partners (as it is, for example, for predators and well-defended prey) then their interests are in line again in that both partners benefit when the interaction is shortened. Such alignment, again, may warrant a certain investment by both partners.

Whenever an interaction involves an encounter between individuals of two species inevitably a common good arises that is shared by these individuals. This is important because, whatever the overall nature of the

Table 1. The effect of changes in demographic rates on the fitness of partners $x$ and $y$, as a function of their respective private-to-common interest ratios $Q_{x}$ and $Q_{y}$. (See Appendix $\mathrm{E}$ for how these weights are derived.)

\begin{tabular}{|c|c|c|c|}
\hline \multirow[t]{2}{*}{ Change } & \multicolumn{2}{|c|}{ Effect on fitness } & \multirow[t]{2}{*}{ Alignment } \\
\hline & $x$ & $y$ & \\
\hline $\begin{array}{l}\text { increase } \sigma_{x} \\
\text { increase } \sigma_{y} \\
\text { increase } \sigma_{x y} \\
\text { decrease } v_{x} \\
\text { decrease } v_{y} \\
\text { decrease } v_{x y} \\
\text { decrease } \delta\end{array}$ & $\begin{array}{l}1 \\
0 \\
Q_{x} \\
Q_{x} \\
Q_{x}-1 \\
Q_{x} \\
Q_{x}-1\end{array}$ & $\begin{array}{l}0 \\
1 \\
Q_{y} \\
Q_{y}-1 \\
Q_{y} \\
Q_{y} \\
Q_{y}-1\end{array}$ & $\begin{array}{l}\text { no } \\
\text { no } \\
\text { always } \\
\text { if } Q_{y}>1 \\
\text { if } Q_{x}>1 \\
\text { always } \\
\text { if } Q_{x}-1 \text { and } Q_{y}-1 \\
\text { of same sign }\end{array}$ \\
\hline
\end{tabular}


interaction, natural selection will always favour at least a minimal investment to bolster the common good. Of course, individuals always have their private interest to cater for as well. Our analysis suggests that when the interaction is of net benefit to the individual, the common interest should be relatively more important, when the interaction is detrimental, private interests should prevail. Yet whichever is the case there is always an optimum balance (Eq. (9)). The concept of common good places such phenomena as reduced virulence in parasites and mutualism in plant-symbiont interactions, and possibly many more that we have not discussed, in a general framework.

Obviously, investment in the common good is a form of cooperation. Interestingly, it is not the only way for cooperation to emerge. Cooperation should result if 'there is something in it' for both partners or, as we call it, when their interests are aligned. It turns out that the alignment of interests follows its own rules. As Table 1 shows, a given interaction may mix a plethora of aligned and conflicting interests. Though the net outcome of the interaction plays a role, there may be alignment in outright competitive interactions just as there will be conflict in mutualistic interactions. Where more than one demographic parameter is affected by a given change (as a consequence of constraints), the outcome may be even more complex. Payoffs may be density and/or state dependent such that cooperation is favoured only at certain times or under certain conditions.

An important insight obtained from this approach is that genetic correlations between partners - the multispecies equivalent of relatedness (Hamilton 1964) which is caused by such mechanisms as vertical transmission and codispersal (Frank 1994, 1996, 1997) - are not a necessary condition for the evolution of cooperation between species, as is often thought (but see GenkaiKato and Yamamura 1999). The mere fact that partners' individual fates are intertwined is enough for a common good to arise. Such a common good is missed in most population dynamical models, as these do not keep track of interacting partnerships. The exception is formed, of course, by host-parasite and host-symbiont models which indeed tend to predict that there should be some common good favouring mutually advantageous traits (such as reduced virulence of parasites) (Hochberg 2000). It is only through the intertwinedness of the fate of both partners that a shared 'extended phenotype' arises (sensu Dawkins 1982). Wilson's (1977) concept of 'trait groups' is also derived from the idea of shared phenotypes.

This does not mean that genetic correlations (vertical transmission, etc.) do not play an important role: such correlations tend to enhance the common good and thus tend to favour cooperation. But the effect may be not so strong for interactions that accrue a net benefit to only one of the partners, such as in host-parasite interactions. For example, when vertical transmission of parasites occurs, parasites should be nicer to their hosts, but the latter should actually increase their effort to fight the parasite. After all, it is not only the infected individual itself that pays the cost, but also its progeniture. Only when the parasite becomes so nice that it should no longer be called a parasite does it pay the hosts to tolerate vertical transmission and cooperate with what now should be called its 'mutualistic symbiont'.

Since such transitions occur when free host fitness becomes less than bound fitness (i.e., when infected hosts fare better than uninfected hosts), this depends not only on the details of the individual interaction, but also on all other aspects of the host-parasite ecology. For example, the evolutionary transition reported by Law and Dieckmann (1998) hinges upon the weaker partner (the host) trading its ability to persist on its own to limit exploitation by its stronger partner. Similar transitions may therefore occur in the presence of other factors that reduce the fitness value of the free-living stage more than that of individuals in association.

A well-known example that fits this idea is that of an avirulent parasite that offers protection against more virulent parasites. For example, some bacterial phages prevent insertion of another phage into their host's genome (Stewart and Levin 1984). Carrying such a phage confers a cost. After all, the phage, even if it does not outright kill its host, remains a parasite that exploits its host to its own end. However, if the risk of infection with much more virulent parasites is sufficiently high, the infection with the avirulent parasites confers a net benefit (it effectively reduces free-living fitness $F_{x}$ ). Thus, also in this case, there is an overlap in interest between the host individual and its parasite that may favour further cooperation.

Many parasites are so weakly virulent that their virulence is difficult to assess and that they go by the name of 'commensalists'. Gut microflora are usually called commensalists, for example. Nevertheless their combined effect may be considerable. An indication is provided by the use of so-called 'growth enhancers' in animal husbandry. It is a widespread practice to mix large amounts of antibiotics through animal feed. The economic reason is increased efficiency of food production (Doluschitz and Zeddies 1991): apparently the gut microflora use up enough resources to stunt their host's growth. If killing the commensals boosts somatic growth by several percent, they are actually parasites. The host's reason to put up with them might be that they offer protection against more virulent parasites. Livestock breeders can get away with killing the commensals, because they can breed their livestock in almost sterile environments, free from more virulent parasites.

The list of examples where former antagonists have merged into single lineages is very long. One might hypothesize that evolution may go backwards as well, 
that is, merged lineages split up in its constituent entities. To our knowledge, there are few examples of such evolutionary retrogressions. It has been hypothesized that fathers do not contribute cell plasm to their offspring to prevent among-strain competition. Such among-strain competition would increase selfish interests of the mitochondria, so that they would contribute less to their carrier's well-being (Godelle and Reboud 1995). Perhaps a better example is that of the endogenous retroviruses (ERVs), viruses that have been captured and 'domesticated' early in the evolution of mammals. These ERVs are dormant normally, but it is thought that they perform an essential function during placenta formation (Löwer et al. 1996, Harris 1998). Thus, they are useful liaisons, but they seem to be dangerous too: ERVs have been associated with various forms of cancers and autoimmune diseases (Löwer et al. 1996, Harris 1998). This would indicate that they have not lost all of their means for horizontal transmission. In another example, a behavioural change that boosted the private interests of an endogenous retrovirus resulted in a significant increase in its virulence (Salemi et al. 1999).

The methods and concepts that we develop in this paper should be particularly useful when interactions are considered whose nature is not immediately obvious. For example, there is evidence for cooperative interactions between some plants and the arthropod predators of the plants' insect herbivores (Takabayashi and Dicke 1996, De Moraes et al. 1998, Sabelis et al. 1999). When such plants are attacked by insect herbivores as aphids or herbivorous mites, they emit an airborne signal that attracts the predators (parasitoids, predatory mites). Can such an interaction be called mutualistic? Of course, both plant and predator benefit if the predators locate their prey. However, there is scope for conflict too: for example, it is in the plants interest if the herbivores are completely exterminated, but to the predators the marginal benefit may eventually drop below a threshold value and they leave. Worse even, under some conditions it may pay the predators to 'milk' their prey population (van Baalen and Sabelis 1995), which is not in the interest of the plant at all.

It has become textbook knowledge that essentially all forms of biological organization are the result of association of lower-level entities. Genes cooperate to form genomes, genomes cooperate with various organelles to form cells, cells cooperate to form multicellular organisms, different organisms cooperate to form symbioses, and so forth.

Yes the very fact that we observe these lower-level entities suggests that they still have some form of independence. And whenever there is such independence, components will sacrifice a bit of the well-being of the whole to promote their private interests. If it is so advantageous to channel reproduction to reduce these interests to reduce conflict (Michod 1996, 1999), why do individuals still put up with sexual reproduction (in which case an 'individual' is actually an association of two gametes)? Maybe it just is because the components (genes, symbionts, etc.) have failed to renounce their evolutionary sovereignty. This is important because it preserves a certain amount of within-organismal conflict. How much of an organism's potential for reproductive output is thus wasted by within-organismal conflicts is difficult to assess. The only way to obtain insight into this question is by assessing carefully how the constituting entities balance their private interests with the common good.

Our framework covers many interactions but is limited in many ways too. Perhaps the most important limitation of the analysis presented here is that it does not take into account the possibility that partners may try to induce a change in the life-history characteristics of their partners. Certain forms of such induced changes are indeed common; parasite-mediated changes in host behaviour are the prime example. Such 'manipulation' complicates the issue, because optimum strategies will then depend on the way partners will respond. With such behavioural flexibility a whole new suite of outcomes may be possible, ranging from simple manipulation via bargaining to fullblown communication between partners. How the balance between common and private interests may affect the evolution of communication strategies will be explored in a separate article.

Acknowledgements - Michael Hochberg, Willem Iemhoff and Yrjö Haila are thanked for their comments. MvB is currently supported by the French Centre National de la Recherche Scientifique but for the research reported in this paper support by the Royal Netherlands Academy of Arts and Sciences (KNAW) is gratefully acknowledged. VAAJ is supported by The Wellcome Trust, grant nr 051319. Both authors acknowledge additional support by the British Council.

\section{References}

Bronstein, J. L. 1994a. Our current understanding of mutualism. - Q. Rev. Biol. 69: 31-51.

Bronstein, J. L. 1994b. Conditional outcomes in mutualistic interactions. - Trends Ecol. Evol. 9: 214-217.

Caro, T. M. 1995. Pursuit deterrence revisited. - Trends Ecol. Evol. 10: 500-503.

Caro, T. M., Lombardo, L., Goldizen, A. W. and Kelly, M. 1995. Tail-flagging and other antipredator signals in whitetailed deer: new data and synthesis. - Behav. Ecol. 6: $442-450$

Cheng, T. C. 1991. Is parasitism symbiosis? A definition of terms and the evolution of concepts. - In: Toft, C. A., Aeschlimann, A. and Bolis, L. (eds), Parasite-host associations - coexistence or conflict? Oxford Univ. Press, pp. $15-36$

Dawkins, R. 1976. The selfish gene. - Oxford Univ. Press.

Dawkins, R. 1982. The extended phenotype. - Freeman.

De Moraes, C. M., Lewis, W. J., Paté, P. W. et al. 1998. Herbivore-infested plants selectively attract parasitoids. Nature 393: $570-573$. 
Dieckmann, U. and Law, R. 1996. The dynamical theory of coevolution: a derivation from stochastic ecological processes. - J. Math. Biol. 34: 579-612.

Doluschitz, R. and Zeddies, J. 1991. Economic effects of the use of Avoparcin in diary cattle feeding. - Ber. Landwirtsch. 69: 365-378.

Frank, S. A. 1994. Kin selection and virulence in the evolution of protocells and parasites. - Proc. R. Soc. Lond. B 258: $153-161$.

Frank, S. A. 1996. Models of parasite virulence. - Q. Rev. Biol. 71: 37-78.

Frank, S. A. 1997. Models of symbiosis. - Am. Nat. 150: S80-S99.

Genkai-Kato, M. and Yamamura, N. 1999. Evolution of mutualistic symbiosis without vertical transmission. Theor. Popul. Biol. 55: 309-323.

Geritz, S. A. H., Metz, J. A. J., Kisdi, E. and Meszéna, G. 1997. Dynamics of adaptation and evolutionary branching. - Phys. Rev. Lett. 78: 2024-2027.

Godelle, B. and Reboud, X. 1995. Why are organelles uniparentally inherited? - Proc. R. Soc. Lond. B 259 27-33.

Haig, D. 1997. The social gene. - In: Krebs, J. R. and Davies, N. B. (eds), Behavioural ecology. An evolutionary approach. 4th ed. Blackwell, pp. 285-304.

Hamilton, W. D. 1964. The genetical evolution of social behaviour. I and II. - J. Theor. Biol. 7: 1-16, 17-52.

Hamilton, W. D. 1967. Extraordinary sex ratios. - Science 156: $477-488$.

Harris, J. R. 1998. Placental endogenous retrovirus (ERV): structural, functional, and evolutionary significance. BioEssays 20: 307.

Heesterbeek, J. A. P. and Metz, J. A. J. 1993. The saturating contact rate in marriage and epidemic models. - J. Math. Biol. 31: 529-539.

Herre, E. A., Knowlton, N., Mueller, U. G. and Rehner, S. A. 1999. The evolution of mutualisms: exploring the paths between conflict and cooperation. - Trends Ecol. Evol. 14: 49-53.

Hochberg, M. E. 2000. A geographical perspective of virulence. - In: Poulin, R., Morand, S. and Skorping, A. (eds), Evolutionary biology of host-parasite relationships: theory meets reality. Elsevier, pp. 81-96.

Hurst, L. D. 1995. Selfish genetic elements and their role in evolution - The evolution of sex and some of what that entails. - Philos. Trans. R. Soc. Lond. B 349: 321-332.

Keller, L. 1999. Levels of selection in evolution. - Princeton Univ. Press.

Kinzig, A. P. and Harte, J. 1998. Selection of micro-organisms in a spatially explicit environment and implications for plant access to nitrogen. - J. Ecol. 86: 831-853.

Kostitzin, V. A. 1934. Symbiose, Parasitisme et Évolution (Étude Mathématique). - Hermann et Cie.

Law, R. and Dieckmann, U. 1998. Symbiosis through exploitation and the merger of lineages in evolution. - Proc. Soc. Lond. B 265: 1245-1253.

Leigh, E. G. 1999. Levels of selection, potential conflicts, and their resolution - the role of the "Common Good". - In: Keller, L., (ed.), Levels of selection in evolution. Princeton Univ. Press, pp. 15-30.

Leigh, E. G. and Rowell, T. E. 1995. The evolution of mutualism and other forms of harmony at various levels of biological organization. - Ecologie 26: 131-158.

Löwer, R., Löwer, J. and Kurth, R. 1996. The viruses in all of us: characteristics and biological significance of human endogenous restrovirus sequences. - Proc. Natl. Acad. Sci. USA 93: 5177.

Margulis, L. 1970. The origin of eucaryotic cells. - Yale Univ. Press.

Maynard Smith, J. 1982. Evolution and the theory of games. - Cambridge Univ. Press.

Maynard Smith, J. and Price, G. R. 1973. The logic of animal conflict. - Nature 246: 15-18.
Maynard Smith, J. and Szathmáry, E. 1995. The major transitions in evolution. - Freeman.

Metz, J. A. J., Nisbet, R. M. and Geritz, S. A. H. 1992. How should we define 'fitness' for general ecological scenarios. Trends Ecol. Evol. 7: 198-202.

Michod, R. E. 1996. Cooperation and conflict in the evolution of individuality. - Proc. R. Soc. Lond. B 263: 813822 .

Michod, R. E. 1999. Individuality, immortality, and sex. - In: Keller, L. (ed.), Levels of selection in evolution. Princeton Univ. Press, pp. 53-74.

Omacini, M., Chaneton, E. J., Ghersa, C. M. and Muller, C. B. 2001. Symbiotic fungal endophytes control insect hostparasite interaction webs. - Nature 409: 78-81.

Parker, M. A. 1999. Mutualism in metapopulations of legumes and rhizobla. - Am. Nat. 153: S48-S60.

Pomiankowski, A. 1999. Intragenomic conflict. - In: Keller, L. (ed.), Levels of selection in evolution. Princeton Univ. Press, pp. 121-152.

Rand, D. A., Wilson, H. B. and McGlade, J. M. 1994 Dynamics and evolution: evolutionarily stable attractors, invasion exponents and phenotype dynamics. - Philos. Trans. R. Soc. Lond. B 343: 261-283.

Sabelis, M. W, van Baalen, M., Bakker, F. M. et al. 1999. The evolution of direct and indirect defence against herbivorous anthropods. - In: Olff, H., Brown, V. and Drenth, R. H. (eds), Herbivores: between plants and predators. Blackwell, pp. $109-166$

Salemi, M., Lewis, M., Egan, J. F. et al. 1999. Different population dynamics of human $\mathrm{T}$ cell lymphotropic virus type II in intravenous drug users compared with endemically infected tribes. - Proc. Natl. Acad. Sci. USA 96: $13253-13258$.

Sapp, J. 1994. Evolution by association: a history of symbiosis. - Oxford Univ. Press.

Stewart, F. M. and Levin, B. R. 1984. The population biology of bacterial viruses: why be temperate. - Theor. Popul. Biol. 26: 93-117.

Takabayashi, J. and Dicke, M. 1996. Plant-carnivore mutualism through herbivore-induced carnivore attractants. Trends Plant Sci. 1: 109-113.

van Baalen, M. and Sabelis, M. W. 1995. The milker-killer dilemma in spatially structured predator-prey interactions. - Oikos 74: 391-400.

Wilson, D. S. 1977. Structured demes and the evolution of group advantageous traits. - Am. Nat. 111: 157-185.

Wolin, C. L. 1985. The population dynamics of mutualistic systems. - In: Boucher, D. H. (ed.), The biology of mutualism. Oxford Univ. Press, pp. 246-269 (cites Kostitzin 1934, 1937).

Yamamura, N. 1993. Vertical transmission and evolution of mutualism from parasitism. - Theor. Popul. Biol. 44: 95-109.

Yamamura, N. 1996. Evolution of mutualistic symbiosis - a differential equation model. - Res. Popul. Ecol. 38: 211218.

\section{Appendix A. A common ecological framework}

Predator-prey, host-parasite, and client-provider interactions can be derived as limiting cases of the framework given by Eqs (1). The easiest way this can be seen is by time-scale separation.

If the rates of complex formation and dissociation ( $\beta$ and $\delta$ ) are much greater than all other demographic rates in the model, we can assume that the complexes are always in 'pseudo-equilibrium'. That is, even when 
$[x]$ and $[y]$ are changing, $\mathrm{d}[x y] / \mathrm{d} t$ will be approximately zero. If we substitute the resulting pseudo-equilibrium value

$$
\overline{[x y]}=\frac{\beta}{v_{x}+v_{y}+v_{x y}+\delta-\sigma_{x y}}[x][y] \approx \frac{\beta}{\delta}[x][y]
$$

into framework (1) we essentially lose the interaction complex and we obtain the classical Lotka-Volterra equations for two interacting species:

$$
\begin{aligned}
& \frac{\mathrm{d}[x]}{\mathrm{d} t}=[x]\left(\rho_{x}+\sigma_{x} \frac{\beta}{\delta}[y]-\mu_{x}\right) \\
& \frac{\mathrm{d}[y]}{\mathrm{d} t}=[y]\left(\rho_{y}+\sigma_{y} \frac{\beta}{\delta}[x]-\mu_{y}\right) .
\end{aligned}
$$

Note that we allow demographic rates to be density dependent. For example, $\rho_{x}$ and $\rho_{y}$ may be negatively dependent on density. Nevertheless, if $\sigma_{x}$ and $\sigma_{y}$ are sufficiently large to overcome the effect of resource competition and the classical 'ecological' equations for a mutualistic interaction emerge.

However, if the rates of formation and dissociation are comparable to at least some of the other rates we obtain other outcomes. For example, [y] will be in pseudo-equilibrium rather than $[x y]$, with

$\overline{[y]}=\frac{\sigma_{y}+v_{y}+\delta}{\mu_{y}+\beta[x]-\rho_{y}}[x y] \approx \frac{\sigma_{y}}{\mu_{y}}[x y]$

if the rates of production of free $y$ by $x y$-complexes and decay of free $y\left(\sigma_{y}\right.$ respectively $\left.\mu_{y}\right)$ are both higher than the other demographic rates. Then the system is approximated by

$$
\begin{aligned}
& \frac{\mathrm{d}[x]}{\mathrm{d} t}=\rho_{x}[x]+\sigma_{x}[x y]-\mu_{x}[x]-\frac{\beta \sigma_{y}}{\mu_{y}}[x][x y]+\delta[x y] \\
& \frac{\mathrm{d}[x y]}{\mathrm{d} t}=\sigma_{x y}[x y]+\frac{\beta \sigma_{y}}{\mu_{y}}[x][x y]-\left(v_{x}+v_{y}+v_{x y}\right)[x y]-\delta[x y]
\end{aligned}
$$

in which case the standard model for host-microparasite interactions with vertical transmission and recovery is obtained (an infected host is essentially a complex of a host and a parasite individual).

If there is a direct connection between the death of one of the partners, say of $x$, and reproduction of the other $(y)$, for example $v_{x}=v_{x}^{0}+D_{y}$ while $\sigma_{y}=\sigma_{y}^{0}+$ $(1+c) D_{y}$, then the framework describes a predatorprey system. If the rates of association $(\beta)$ and killing $\left(D_{y}\right)$ are sufficiently high, then a classical predator-prey model with type II functional response results.

A 'provider-client' interaction results if complex dynamics is fast ( $\beta$ and $\delta$ large), and if the recruitment of one of the populations, say population $x$, is only carried out by the complex:

$$
\begin{aligned}
& \frac{\mathrm{d}[x]}{\mathrm{d} t}=[x]\left(\sigma_{x} \frac{\beta}{\delta}[y]-\mu_{x}\right) \\
& \frac{\mathrm{d}[y]}{\mathrm{d} t}=[y]\left(\rho_{y}+\sigma_{y} \frac{\beta}{\delta}[x]-\mu_{y}\right) .
\end{aligned}
$$

This kind of relation would arise if plant seeds need to be obligately dispersed by herbivores.

\section{Appendix B. Invasion analysis}

Consider a mutant of population $x$, differing in a trait $\hat{c}_{x}$ that may affect any of its life-history traits (i.e., $\hat{\rho}_{x}=\hat{\rho}_{x}\left(\hat{c}_{x}\right), \hat{\mu}_{x}=\hat{\mu}_{x}\left(\hat{c}_{x}\right), \quad \hat{\sigma}_{x}=\hat{\sigma}_{x}\left(\hat{c}_{x}\right)$, etc.). (Any of these traits may be influenced by the resident's traits as well, but to save ink we will drop this from the notation.)

Adding this mutant in a very small density to the system results in two additional equations, one for the density of free mutant $(\hat{x})$ and one for the mutant in association with a member of the $y$ population $(\hat{x} y)$ :

$\frac{\mathrm{d}[\hat{x}]}{\mathrm{d} t}=\hat{F}_{x}[\hat{x}]-\hat{\beta}[\hat{x}][y]+\hat{P}_{x}[\hat{x} y]$

$\frac{\mathrm{d}[\hat{x} y]}{\mathrm{d} t}=\beta[\hat{x}][y]-\hat{M}_{x y}[\hat{x} y]$.

The mutant is assumed to be so rare as not to affect the dynamics of the resident populations $x$ and $y$. If it is assumed, in addition, that the resident populations are in equilibrium (leading to $[y]=\overline{[y]}$ given by the equilibrium of System (1)), System (15) will be linear and can be written as

$\frac{\mathrm{d}}{\mathrm{d} t}\left(\begin{array}{c}{[\hat{x}]} \\ {[\hat{x} y]}\end{array}\right)=\left(\begin{array}{cc}\hat{F}_{x}-\hat{\beta} \overline{[y]} & \hat{P}_{x} \\ \hat{\beta}[y] & -\hat{M}_{x y}\end{array}\right)\left(\begin{array}{c}{[\hat{x}]} \\ {[\hat{x} y]}\end{array}\right)$

As a consequence, it is easy to calculate the dominant eigenvalue $\hat{\lambda}_{x}$, i.e., the invasion exponent of mutant $\hat{x}$.

It can be shown (see Law and Dieckmann 1998) that if the mutant is close to the resident,

$$
\begin{aligned}
\hat{\lambda}_{x} & =\frac{-\left(\hat{F}_{x}-\hat{\beta} \overline{[y]}\right) \hat{M}_{x y}-\hat{\beta} \overline{[y]} \hat{P}_{x}}{\hat{T}_{x}} \\
& =\left(\frac{-\hat{M}_{x y}}{\hat{T}_{x}}\right)\left(\hat{F}_{x}+\hat{\beta}\left[\overline{[y]} \frac{\hat{P}_{x}-\hat{M}_{x y}}{\hat{M}_{x y}}\right)\right.
\end{aligned}
$$

where $\hat{T}_{x}$ is the trace of the matrix in Eq. (16). Since $\hat{T}_{x}$ will be negative, the sign of the invasion exponent is the same as that of the second factor, which leads to the fitness measures expressed in Eqs (6) and (7). 


\section{Appendix C. Common defence}

As an example of the relativity of the concepts of competition, antagonism and mutualism, consider the following setting. There are two populations whose niches overlap but not completely. According to the ecological definitions, they are competitors. Suppose now that these species share a common predator to which free individuals have no defence but to which associations are protected.

Free individuals have a rate of reproduction $\rho_{i}=$ $r_{i}\left(1-\kappa_{i x}([x]+[x y\})-\kappa_{i y}([y]+[x y])\right)$ and a mortality rate of $\mu_{i}=\mu_{i}^{0}+p$ where $p$ is the predation pressure and $\mu_{i}^{0}$ is the background mortality rate of species $i(i=x$, $y$ ). Bound individuals pay a cost $C_{i}$ terms of reduced reproduction, i.e., $\sigma_{i}=\left(1-C_{i}\right) \rho_{i}$; no joint reproduction is assumed in this example $\left(\sigma_{x y}=0\right)$. Background mortality is the same for bound individuals, $v_{i}=\mu_{i}^{0}$. The complex, however, offers protection against the predators, so that the predation rate (acting on the complex) is reduced by a factor $B$, i.e., $v_{x y}=(1-B) p$.

The nature of the interaction between species $x$ and $y$ is given by the signs of bound fitness, $P_{x}-M_{x y}$ and $P_{y}-M_{x y}$, respectively. (At equilibrium, $F_{i}$ and $P_{i}-$ $M_{x y}$ will have opposite signs, see Fig. 2.) The population-level definitions are derived from the signs of the elements of the community matrix. These elements express the effect of an increase of one species on the mean per capita rate of reproduction (i.e., mean fitness) of another. For our model, therefore, the interaction coefficient of species $y$ on species $x$ is given by $\alpha_{x y}$ where

$\alpha_{x y} \propto \frac{\mathrm{d} W_{x}}{\mathrm{~d}[y]}$

evaluated at equilibrium. With the definitions of the model for common defence this becomes

$\alpha_{x y} \propto \frac{\mathrm{d} F_{x}}{\mathrm{~d}[y]}-\beta \frac{\hat{P}_{x}-\hat{M}_{x y}}{\hat{M}_{x y}}-\beta[y] \frac{\frac{\mathrm{d} \hat{P}_{x}}{\mathrm{~d}[y]}-\frac{P_{x}}{M_{x y}} \frac{\mathrm{d} \hat{M}_{x y}}{\mathrm{~d}[y]}}{\hat{M}_{x y}}$

(Note that we assume that birth rates are density dependent, so that $F_{x}, P_{x}$ and $M_{x y}$ will depend on $[y]$.) We used this relation to draw Fig. 2C.

\section{Appendix D. Mutually assisted competition}

Consider an environment harbouring plants and soil microorganisms as free-living individuals (with densities of $[x]$ and $[y])$ and as plant-microorganism associations (with density $[x y]$ ). For the sake of argument, count the microorganisms that are associated with a particular plant as a single 'individual' (that is, each plant is associated with at most one microorganism clone). The basic idea is that individuals (plants, clones) may trade a bit of their potential for reproduction to help their partner to compete, for example, by exchanging vital resources (Kinzig and Harte 1998, Parker 1999, Hochberg 2000). For clarity, we will assume that there is no joint reproduction $\left(\sigma_{x y}=0\right)$ and no correlated mortality $\left(v_{x y}=0\right)$. That is, there are no factors involved that induce an obvious common interest. Since plants and microorganisms do not move, we assume $\delta=0$.

Under what conditions does it pay an individual of species $x$ to sacrifice a proportion $c_{x}$ of its potential reproductive output to help its partner compete? For an individual of species $x$, the private-to-common interest ratio can be expressed as

$Q_{x}=\frac{P_{x}}{M_{x y}}=\frac{[\text { reproduction }]+[\text { death of } \text { partner }]}{[\text { death of } \text { self }]+[\text { death of } \text { partner }]}$

Investment to reduce reproduction $\left(\sigma_{x}\right)$ in favour of the partner's survival $\left(v_{y}\right)$ is therefore selected if the reduction in private interests

$\frac{\mathrm{d} P_{x}}{\mathrm{~d} c_{x}}=\frac{\mathrm{d}}{\mathrm{d} c_{x}}$ [reproduction] $+\frac{\mathrm{d}}{\mathrm{d} c_{x}}$ [death of partner]

(which is negative as investment both reduces reproduction and partner mortality) is outweighed by the increase in common good

$\frac{\mathrm{d} M_{x y}}{\mathrm{~d} c_{x}}=\frac{\mathrm{d}}{\mathrm{d} c_{x}}$ [death of partner]

(which is positive, as mortality decreases with investment) according to Condition (9), leading to

$\left(Q_{x}-1\right) \frac{\mathrm{d}}{\mathrm{d} c_{x}}$ [death of partner] $<\frac{\mathrm{d}}{\mathrm{d} c_{x}}$ [reproduction].

From this we can conclude that it may pay to decrease your partner's mortality provided that (1) the association is profitable ( $Q_{x}$ larger than one) and (2) the efficacy of the investment is large enough.

For $Q_{x}$ to be larger than unity, it is required that the partner invests in the association as well (if the partner does not reciprocate, the mortality rate of the $x$-individual will be the same as that for free-living individuals and, as a consequence, $Q_{x}$ will not exceed one). Thus, it pays to help your partner but only if your partner helps you. Indeed, Fig. 4 shows that evolutionarily bistability is possible.

Competition (each population for its own resource) is assumed to increase mortality rate of free-living individuals with a factor $K_{i}=\kappa_{i}([i]+[x y])$, so that $\mu_{i}=\mu_{i}^{0}+$ 
$K_{i}$. Exchange of resources with a partner allows to alleviate the effects of competition: if partner $y$ donates a proportion $c_{y}$ of its reproductive output $\rho_{y}$ to $x$, x's sensitivity to competition is reduced by a factor $h_{x}\left(\rho_{y}\right)$ $\left(0<h_{x}\left(\rho_{y}\right)<1\right)$ so that mortality rate in association becomes $v_{x}=\mu_{x}^{0}+h_{x}\left(c_{y} \rho_{y}\right) K_{x}$. For alleviation of competition we took $h_{i}(\rho)=\left(1+\varepsilon_{i} \rho\right)^{-1}$. The costs are expressed in terms of reduced birth rates, i.e., $\sigma_{i}=\left(1-c_{i}\right) \rho_{i}$.

\section{Appendix E. Alignment of interests}

Consider a trait $c_{x}$ that is expressed only in association and that is favoured by natural selection. That is, increasing this trait increases $Q_{x}=P_{x} /\left(M_{x y}\right)$. Denoting the differential with respect to $c_{x}$ by a prime, for such a trait it holds

$P_{x}^{\prime}-Q_{x} M_{x y}^{\prime}>0$.

Demographic rates have different effects on these components. Expanding the differential $P_{x}^{\prime}$ and $M_{x y}^{\prime}$ shows how to 'weigh' their effects:

$\sigma_{x}^{\prime}+v_{y}^{\prime}+\delta^{\prime}-Q_{x}\left(v_{x}^{\prime}+v_{y}^{\prime}+v_{x y}^{\prime}+\delta^{\prime}-\sigma_{x y}^{\prime}\right)>0$.
Grouping the effects by weight yields

$\sigma_{x}^{\prime}+Q_{x}\left(\sigma_{x y}^{\prime}-v_{y}^{\prime}+v_{x y}^{\prime}\right)+\left(Q_{x}-1\right)\left(-v_{y}^{\prime}-\delta^{\prime}\right)>0$.

This shows that (in absence of constraints) increases in $\sigma_{x}$ and $\sigma_{x y}$ are always favoured, as well as decreases in $v_{x}$ and $v_{x y}$. Decreases in $v_{y}$ or $\delta$ are favoured only when the association confers a net benefit $\left(Q_{x}>1\right)$.

Suppose now that the mutant changes its trait in the beneficial direction. What will be the consequences for its partner? This is captured by the resulting change in $Q_{y}=P_{y} / M_{x y}$. The change by $x$ is therefore beneficial for $y$ if

$\sigma_{y}^{\prime}+v_{x}^{\prime}+\delta^{\prime}-Q_{y}\left(v_{x}^{\prime}+v_{y}^{\prime}+v_{x y}^{\prime}+\delta^{\prime}-\sigma_{x y}^{\prime}\right)>0$,

which can be rewritten as

$\sigma_{y}^{\prime}+Q_{y}\left(\sigma_{x y}^{\prime}-v_{y}^{\prime}+v_{x y}^{\prime}\right)+\left(Q_{y}-1\right)\left(-v_{x}^{\prime}-\delta^{\prime}\right)>0$.

How a change in a given parameter affects both partners simultaneously is summarized in Table 1. 